\title{
Effect of post-activation potentiation by combining heavy squats and sprints on the countermovement long jump (Pilot study)
}

\author{
Tereza Králová', Marian Vanderka'2, Jan Cacek', Matěj Matura' , Filip Popelka' \\ ${ }^{1}$ Faculty of sports studies, Masaryk university \\ ${ }^{2}$ Faculty of Physical Education and Sports, Comenius University in Bratislava
}

\begin{abstract}
Weightlifting heavy load exercises are commonly used as a key instrument for enhancing performance if compared to those not using the exercise stimulating muscle tone. Studies examined the post-activation effect (PAP) using only one parameter but not in consecutive and intermingling exercises to prolongated the time of the PAP effect duration and maintain increased performance. Nine male students, with resistance training experience, performed warm-up and exercises enhancing muscle tone (EEMT) using squats to $90^{\circ}$ flexion in the knee joint with $80 \% 1$ RM (repetition maximum) in 3 sets with 3 repetitions of squat in each set (2-3 min. rest intervals). After EEMT they performed a 10-m sprint test in $2^{\text {nd }}$ and $5^{\text {th }}$ min, the countermovement long jump (CLJ) test in the $5^{\text {th }}$ and $6^{\text {th }}$ minute, a 10-m sprint test in the $10^{\text {th }}$ and $11^{\text {th }}$ minute and the CLJ test in the $12^{\text {th }}$ and $14^{\text {th }}$ minute. The results showed that the average improvement was in 10-m sprint after EEMT 0,013 $\pm 0,05$ seconds, but there were no statistical differences observed between the result without and with EEMT $(p>0.05)$. We observed a significant improvement between jumps without and with EEMT in $5-14^{\text {th }}$ min $(p<0.05)$. There were no statistical differences between the results in CLJ carried out in 5-6 th $^{\text {min }}$ after EEMT and after two 10-m sprints and the results carried out in 12-14 ${ }^{\text {th }}$ min. after EEMT, four 10-m sprints and two jumps $(p>0.05)$. It was found that 10-m sprint has no negative effect on performance in the long jump and could be regularly applied to prolong the PAP effect.
\end{abstract}

Keywords: PAP, squat, toning, sprint, jumping

\section{INTRODUCTION}

The performance based on a strength-power ability can be enhanced with muscle pre-toning using weightlifting exercise with heavy loads (Mitchell \& Sale, 2011). The principles of enhancing the performance are usually explained as a post-activation potentiation effect (PAP). The PAP effect is usually induced by a voluntary conditioning contraction and performed typically at a maximum or near-maximal intensity (Anthony \& Bishop, 2009). The mechanism is still not deeply understood, but most researchers believe that the mechanism behind PAP is the phosphorylation of myosin regulatory light chains, which increases the sensitivity of the myofilaments to calcium ions $\left(\mathrm{Ca}^{2+}\right)$ (Grange, Vandenboom, \& Houston, 1993). The PAP is probably sex- and age-dependent, since the PAP effect most occurs in men compared to women (Arabatzi et al., 2014), but recent studies focus on women declared a possible usage for woman as well (Ah Sue, Adams, \& DeBeliso, 2016; Pääsuke et al., 2007). Many studies examine the effect of muscle pre-toning using heavy loads exercises on speed - power - ability movements, but in practice (e.g. athletics or track and field performance) it is not common to use Olympic bars with plates to enhance performance during the competition. Particularly for collective sports training, there is often included a special resistance training containing heavy squats (maximum flexion in the knee joint is $90^{\circ}$ ) to improve the sensibility to PAP (Ah Sue et al., 2016; Sánchez Moreno, García Asencio, \& González Badillo, 2014). But there are also studies, which do not prove any improvement in vertical jump after sets 
of squats in team sports (Birch, Robinson, Nelson, \& Oranchuk, 2017). The main benefit after heavy squats in warm-up training with consecutive sprints and jumps can probably prolong the ability to incite a better performance. This option (combining more factors to prolong the PAP effect) has not yet been investigated.

The aim of this study was to observe and examine how we could prolong the PAP effect duration after multi-sets of heavy loads squats to $90^{\circ}$ flexion in the knee joint through the using of $10-\mathrm{m}$ sprints and the countermovement long jumps on the performance in these exercises.

\section{METHODS}

\section{Subjects}

Nine males (mean age $22.56 \pm 1.42$ years; body mass $74,78 \pm 5.09 \mathrm{~kg}$; body height $180,78 \pm 6,96 \mathrm{~cm}$ ) volunteered to participate in this study after random selection from students with the physical education as a field of study. All participants were students of faculty of sports studies and did not focus only on one sport, but everyone had at least 2 years' experience with resistance training. Their history of injuries did not include any lower-limb or back injury in the previous three years. Before they participated, they went through a school of the squat with a professional weightlifting coach to learn a proper and safe squat technique. All students agreed to participate in the research by signing the informed consent form.

\section{Study design}

This study focused on comparing the performance in 10-m sprint and countermovement long jump with and without exercises enhancing muscle tone (EEMT) by heavy loads squats using patterns of the post-activation potentiation effect. The main purpose was to observe the changes caused due to the successive interaction of the 10-m sprints and countermovement long jump $(\mathrm{CLJ})$ on their results.

Two weeks before the testing, all participants underwent a training session with a professional weightlifting coach. The coach taught the participants a proper and safe technique of squat following these principles: stand position should be comfortable, feet approximately under the hips (or slightly wider) and turned out. Technique - high bar position. During the squat, the pelvis starts to move back and down with the body. The head and the back are held in a neutral upright position. The velocity of an eccentric phase of the squat should be moderate, the concentric phase should be accelerated if possible. During this session, we measured individually the $90^{\circ}$ flexion in the knee joint - as a limit for the squat depth.

One week before testing the participants were measured in the pre-tests on the best results in the 10-m sprint and CLJ, with the same warm-up protocol which we used for the testing protocol. The warm-up protocol included a 5-minute workout with a light load on a stationary bike, 2 minutes of full-body joint mobilization, five exercises of dynamics stretching focused on lower limbs in 4 minutes and then five exercises of running alphabet per $10 \mathrm{~m}$. After the warm-up protocol, the testing of 10-m sprint and the CLJ, the one repetition maximum in a squat with $90^{\circ}$ flexion in the knee joint was determined. All participants wore a classic sports clothing for physical education lesson and the indoor type of shoes.

The Testing protocol (Fig. 1) had the following time management: warm-up protocol, muscle toning using squats in $3 \times 3$ with $80 \%$ of $1 \mathrm{RM}$ (repetition maximum) with rest intervals $2-3$ min, $10-\mathrm{m}$ sprint test in the $2^{\text {nd }}$ and $4-5^{\text {th }}$ minute after the end of squats, in the $5^{\text {th }}$ and $6^{\text {th }}$ minute the CLJ test, in the $10^{\text {th }}$ and $11^{\text {th }}$ minute $10-\mathrm{m}$ sprint test and in the $12^{\text {th }}$ and $14^{\text {th }}$ minute CLJ test. The intensity of the squat in this study was established regarding the fact of safety load squats 
compared to participants experience with this exercise. Recent studies show that optimal intensity could be almost $87 \%$ for squat in three repetitions to observe an increase in jump performance (Poulos et al., 2018).

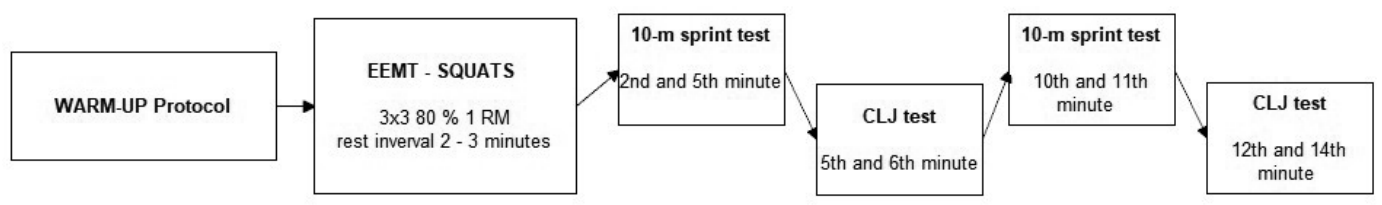

Fig. 1 Testing protocol

\section{THE TESTING PROCEDURES}

\section{Sprint testing}

The 10-m sprint test was used as a test of acceleration and sprint ability. For the time measuring we used the photocells timing system by TVR Elektronik. The test was carried out in a hall with a stable temperature. The testing track was limited by two white lines on the floor. Participants started $50 \mathrm{~cm}$ behind the first photocell from the white $5 \mathrm{~cm}$ wide line to eliminate measurement errors (Thomas, Jones, Rothwell, Chiang, \& Comfort, 2015). Start position was the relay start position (with one hand on the floor). Each participant performed two trials with one-minute rest between the trials. The best performance from these two trials in each specified time was used for further analysis as a pre-test.

\section{Countermovement long jump (CLJ) testing}

Countermovement long jumps were performed with the hands on the hips to eliminate the involvement of hands. The participant stood behind the starting line, with feet comfortably on the pelvic width apart and pushed off with maximum effort and jumped forward as far as possible. The distance was measured from the take-off line to the point where the back of the heel nearest to the take-off line lands on the floor (Castro-Piñero et al., 2010). The test was measured one minute after the $10-\mathrm{m}$ sprint test and repeated twice in the specified time (in $2^{\text {nd }}$ and $5^{\text {th }}$ minute; $12^{\text {th }}$ and $14^{\text {th }}$ minute). The best score was selected for analyses (in $\mathrm{cm}$ ).

\section{RM squat testing}

The one repetition maximum in a full squat with $90^{\circ}$ flexion in the knee joint determined using Stopanni (2008) Testing 1 RM protocol. After an assessment of 1 RM, using Brzycki (Brzycki, 1993) protocol for counting the $80 \%$ of 1 RM individually for each participant (Tab.1) was taken. This test was controlled by the weightlifting professional coach to observe and supervise this testing to comply with safety principles and proper technique of squats.

Tab. 1: Assessing the testing loads for the toning squats

\begin{tabular}{|l|c|c|c|c|c|c|c|c|c|}
\hline Participant & P 1 & P 2 & P 3 & P 4 & P 5 & P 6 & P 7 & P 8 & P 9 \\
\hline Load (kg)/ repetition & $70 \times 5$ & $85 \times 2$ & $105 \times 5$ & $125 \times 7$ & $100 \times 5$ & $73 \times 2$ & $85 \times 2$ & $145 \times 1$ & $115 \times 3$ \\
\hline 1 RM (kg) & 79 & 87 & 118 & 150 & 113 & 75 & 87 & 145 & 122 \\
\hline 80 \% z 1 RM (kg) & 63 & 70 & 94 & 120 & 90 & 60 & 70 & 116 & 98 \\
\hline
\end{tabular}




\section{Strength protocol of the exercises enhancing muscle tone (EEMT)}

Strength exercises stimulate the emergence of the PAP effect more in multiple sets, longer recovery intervals and shallower depth of squat (to $90^{\circ}$ degrees in comparison to deep squat under $90^{\circ}$ ) in effect on sprinting and jumping (Seitz \& Haff, 2016). For stimulating the PAP effect we used three multiple sets with three repetitions in each set of squats with a load corresponding to $80 \%$ of one repetition maximum. EEMT protocol consisted of the squats up to $90^{\circ}$ of knee flexion. Interval of recovery was 3 minutes after each attempt:

1. Squats with body weight $1 \times 3$.

2. Squats with $20 \mathrm{~kg}$ Olympic bar $1 \times 3$.

3. Squats with $60 \%$ of $1 \mathrm{RM} 1 \times 3$.

4. EEMT: Squats: 3 sets with $80 \%$ of $1 \mathrm{RM}$ with 3 repetitions in each set.

\section{STATISTICAL ANALYSES}

All statistical analyses were performed using a program STATISTICA 12. For the statistical analysis, we used a t-test, an analysis of variance (ANOVA) and repeated measures ANOVA and the level of significance was set at $\mathrm{p}<0.05$.

\section{RESULTS}

The results were statistically analyzed separately. In the 10-m test, we put together the results of $10-\mathrm{m}$ sprint after EEMT in $2^{\text {nd }}-11^{\text {th }}$ minute and the best results from all trials from each participant were compared to results in a 10-m sprint without EEMT. The average improvement was $0.013 \pm 0.05$ seconds in 10-m sprit after EEMT, but there were no statistical differences observed between the result without and with EEMT ( $p>0.05)$.

In the countermovement long jump with the elimination of upper limb help, we put together the best results after EEMT and we compared them with the results without EEMT, the results achieved in $5^{\text {th }}$ and $6^{\text {th }}$ min. after EEMT and the results achieved in $12^{\text {th }}$ and $14 \mathrm{t}^{\mathrm{h}}$ min. after EEMT (Tab. 2).

Tab. 2: Countermovement long jump results

\begin{tabular}{|l|c|c|c|c|}
\hline Variable & Average & Minimum & Maximum & Standard deviation (SD) \\
\hline Age (years) & 22.56 & 21.00 & 25.00 & 1.42 \\
\hline Body weight $(\mathrm{kg})$ & 74.78 & 69.00 & 82.00 & 5.09 \\
\hline Body height (cm) & 180.78 & 172.00 & 192.00 & 6.96 \\
\hline 1 RM (kg) & 108.44 & 75.00 & 150.00 & 27.98 \\
\hline Jump without pre-EEMT (cm) & 215.83 & 193.00 & 234.00 & 11.56 \\
\hline Jump in 5.-14. min. after EEMT (cm) & 224.61 & 199.00 & 235.00 & 12.10 \\
\hline Jump in 5.-6. min. after EEMT (cm) & 221.28 & 195.00 & 235.00 & 12.90 \\
\hline Jump in 12.-14. min. after EEMT (cm) & 220.39 & 199.00 & 235.00 & 13.25 \\
\hline
\end{tabular}

We observed significant improvement between jumps without and with multiple sets of highintensity weightlifting exercise in $5-14^{\text {th }} \min (\mathrm{p}<0.05)$. There were no statistical differences between the results carried out in $5-6^{\text {th }}$ min after sets of squats and after two 10-m sprints and the results carried out in $12-14^{\text {th }} \mathrm{min}$. after sets of squats, four 10-m sprints and two jumps (Fig. 2). 


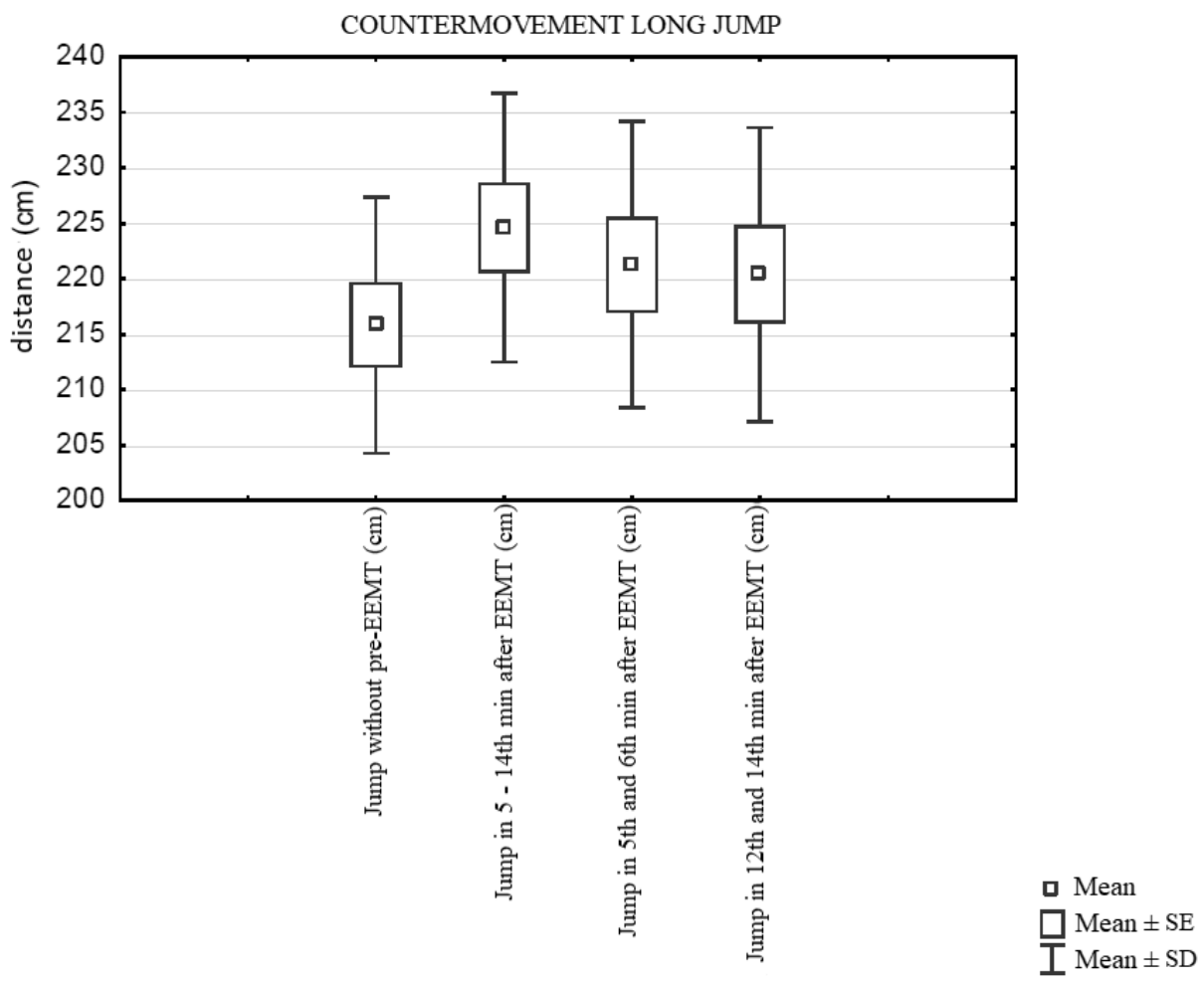

Fig. 2 Countermovement long jump results ( $S D=$ standard deviation, $S E=$ standard error of the mean)

\section{DISCUSSION}

This study examined the influence of combining sprint and jump after heavy squats to prolong the PAP effect duration. It also examined the performances in the 10-m sprint and CLJ according to rest intervals. The main findings in this study are 1) no significant changes in performance in 10 -m sprint with and without EEMT using heavy squats $(3 \times 380 \% 1 \mathrm{RM}) 2)$ positive relationship of EEMT using heavy squats $(3 \times 380 \% 1 \mathrm{RM})$ to countermovement long jump performance 3$)$ no significant differences between the CLJ performance conducted in 5. and 6. min compared to performance in CLJ conducted in $12^{\text {th }}$ and $14^{\text {th }}$ min. after EEMT 4) sprint running did not cause any decrease in CLJ performance.

The experimental protocol of this study was designed to achieve the PAP effect on 10-m sprints encouraging the PAP effect in CLJ. We established the partial components considering the similarity in the biomechanical pattern between the half squat and start sprint position and CLJ movement. The similarity in the biomechanical patterns was based on the fact that the knee joint angle during the squat was minimum at $90^{\circ}$ of the knee flexion as similar to relay start position using in the 10-m sprint test and also the nearly same knee angle as the deepest position for CLJ without upper limb movement. The high intensity of $80 \% 1 \mathrm{RM}$ in the squat was sufficient to cause the PAP effect in the subsequent exercises and movements (Fukutani et.al., 2014).

We hypothesized that we could enhance the jump performance by multiple sets of squats with adequate intensity and prolong the PAP effect duration using the sprint exercise. We tested the hypothesis if the 10-m sprint did not negatively cause a decrease of jump exercise. Our results showed, that the performance in CLJ was significantly increased by multiple sets of squats in 
comparison to the performance without using the EEMT. We hypothesized that sprinting could have the stimulating effect to stay ready for executing jump performance, still with potentiation effect from EEMT using the heavy squats.

There were four potential limitations to consider in this study. First, a small number of participants and their daily training schedule, even though we planned the muscle pre-testing and testing sessions with sufficient distance from their physical activities. Second, the PAP effect should be small for a jump and moderate for a sprint performance activity (Seitz \& Haff, 2016), but in this study, there was not found an effect on sprint performance, but we found the effect for CLJ. The third, the rest intervals could be larger to enhance more the PAP effect. The change of the rest intervals could completely change the results. The fourth, the type of warm-up protocol. In this study, we used the traditional warm-up strategy, which included low loaded endurance exercises and sport specific drills and did not include the warm-up incorporating PAP. All the limitations mentioned seems to be an initiative for other studies to examine the larger spectrum of rest intervals to simulate the competition conditions better.

\section{CONCLUSION}

The present study shows, that combining the EEMT at high intensity with sprints can be used to prolong the PAP effect on jump performance. The multiple sets of squats are helpful to enhance the jump performance. In a real competition at the national level, the competitors mostly have a chance to go to the gym (or the special places such as weightlifting area) and carry out the high-intensity weightlifting exercise. After the multiple sets of high-intensity weightlifting exercise, there could be enough time when the athletes could promote this increased potentiation and prolong the PAP effect. We assume that sprinting can be the exercise which could help the athletes maintain increased neuro-muscle activity, which leads to better performance.

\section{References}

Ah Sue, R., Adams, K., \& DeBeliso, M. (2016). Optimal Timing for Post-Activation Potentiation in Women Collegiate Volleyball Players. Sports, 4(2), 27. https://doi.org/10.3390/sports4020027

Anthony, N., \& Bishop, D. (2009). Factors Modulating Post-Activation Potentiation and its effects on performance. Sports Medicine, 39(2), 147-166. https://doi.org/10.1007/s00221-005-0274-9

Arabatzi, F., Patikas, D., Zafeiridis, A., Giavroudis, K., Kannas, T., Gourgoulis, V., \& Kotzamanidis, C. M. (2014). The PostActivation Potentiation Effect on Squat Jump Performance: Age and Sex Effect. Pediatric Exercise Science, 26(2), 187-194. https://doi.org/10.1123/pes.2013-0052

Birch, E. W., Robinson, T. L., Nelson, M. C., \& Oranchuk, D. J. (2017). Original Scientific Research Study Neither Supra-Maximal Rack-Squats nor Moderately Loaded Jump-Squats Elicit Post-Activation Potentiation in NCAA Divison II Volleyball and American Football Players. Journal of Australian Strength \& Conditioning, 25(3), 20-69.

Brzycki, M. (1993). Strength Testing-Predicting a One-Rep Max from Reps-to-Fatigue. Journal of Physical Education, Recreation \& Dance, 64(1), 88-90. https://doi.org/10.1080/07303084.1993.10606684

Castro-Piñero, J., Ortega, F. B., Artero, E. G., Girela-Rejón, M. J., Mora, J., Sjöström, M., \& Ruiz, J. R. (2010). Assessing muscular strength in youth: Usefulness of standing long jump as a general index of muscular fitness. Journal of Strength and Conditioning Research, 24(7), 1810-1817. https://doi.org/10.1519/JSC.0b013e3181ddb03d

Fukutani, A., Takei, S., Hirata, K., Miyamoto, N., Kanehisa, H., \& Kawakami, Y. (2014). Influence of the intensity of squat exercises on the subsequent jump performance. The Journal of Strength \& Conditioning Research, 28(8), 2236-2243.

Grange, R. W., Vandenboom, R., \& Houston, M. E. (1993). Physiological Significance of Myosin Phosphorylation in Skeletal Muscle. Canadian Journal of Applied Physiology, 18(3), 229-242. https://doi.org/10.1139/h93-020

Mitchell, C. J., \& Sale, D. G. (2011). Enhancement of jump performance after a 5-RM squat is associated with postactivation potentiation. European Journal of Applied Physiology, 111 (8), 1957-1963. https://doi.org/10.1007/s00421-010-1823-x

Pääsuke, M., Saapar, L., Ereline, J., Gapeyeva, H., Requena, B., \& ööpik, V. (2007). Postactivation potentiation of knee extensor muscles in power- and endurance-trained, and untrained women. European Journal of Applied Physiology, 101(5), 577-585. https://doi.org/10.1007/s00421-007-0532-6 
Poulos, N., Chaouachi, A., Buchheit, M., Slimani, D., Haff, G. G., Newton, R. U., \& Germain, P. Saint. (2018). Complex Training and Countermovement Jump Performance Across Multiple Sets : Effect of Back Squat Intensity. Kinesiology, 50, 75-89.

Moreno, M. S., Asencio, C. G., \& Badillo, J. J. G. (2014). The effects of short-term resistance program on vertical jump ability in elite male volleyball players during the competition season. Retos: nuevas tendencias en educación física, deporte y recreación, (26), 153-156.Seitz, L. B., \& Haff, G. G. (2016). Factors Modulating Post-Activation Potentiation of Jump, Sprint, Throw, and Upper-Body Ballistic Performances: A Systematic Review with Meta-Analysis. Sports Medicine, 46(2), 231-240. https://doi.org/10.1007/s40279-015-0415-7

Thomas, C., Jones, P. a., Rothwell, J., Chiang, C.-Y., \& Comfort, P. (2015). An Investigation Into the Relationship Between Maximum Isometric Strength and Vertical Jump Performance. Journal of strength and conditioning research, 29(8), 2176-85. https://doi.org/10.1519/JSC.0000000000000866

Stoppani, J. (2008). Velká kniha posilování. Grada Publishing as.

Wirth, Klaus., Hartmann, Hagen., Sander, Adre., Mickel, Christopn., Szilvas, Elena., and Keiner, M. (2016). The Impact of Back Squat and Leg-Press Exercises on Maximal Strength and Speed-Strength Parameters. Journal fo Strength and Conditioning Research, 30(5), 1205-1212. https://doi.org/10.1519/JSC.0000000000001228 